\title{
Photochemical Kinetics of Maleic to Fumaric Acid on Silver Nanoparticle Surfaces
}

\author{
Nak Han Jang, ${ }^{*}$ Dae Hong Jeong, ${ }^{\dagger}$ and Jung Sang Suh ${ }^{\ddagger, *}$ \\ Institute of Science Education, Kongju National University, Kongju, Chungnam 314-701, Korea. *E-mail: nhjang@kongju.ac.kr \\ ${ }^{\dagger}$ Department of Chemistry Education, Seoul National University, Seoul 151-742, Korea \\ "School of Chemistry and Molecular Engineering, Seoul National University, Seoul 151-742, Korea \\ *E-mail: jssuh@plaza.snu.ac.kr \\ Received February 17, 2005
}

\begin{abstract}
A visible photochemistry of maleic to fumaric acid adsorbed on silver nanoparticle surfaces was investigated as probed by SERS using a simple flow method. Photoisomerization of maleic to fumaric acid was consecutively observed in the condition of various flow rates, which varied the exposure time of laser beam. The sequential SERS spectra of maleic acid indicated that the photochemical isomerization and desorption took place simultaneously on silver nanoparticle surfaces as a function of laser fluency and wavelength. For 530.9 $\mathrm{nm}$ laser line excitation, the rate constant coefficients were obtained with $a=5.9 \mathrm{sec}^{-1} \mathrm{~mW}$ for isomerization and $b=13.9 \mathrm{sec}^{-1} \mathrm{~mW}$ for desorption, which $k_{1}=a \mathrm{I}^{\mathrm{n}}$ and $k_{2}=b \mathrm{I}^{\mathrm{m}}$. Both reactions were one photon process $(n$ $=1, m=1$ ) of a visible light and relatively fast process whose decay time was in the range of milli-second for $50 \mathrm{~mW}$ laser power. The rate of photochemical reaction increased on going toward the blue and photodesorption was a dominant process. A simple flow method used in this study was very useful to study a relatively fast photochemical reaction of molecules adsorbed on silver nanoparticle surfaces.
\end{abstract}

Key Words : Photochemistry, Kinetics, Maleic acid, Fumaric acid, Silver nanoparticles

\section{Introduction}

The study of photochemistry at surfaces and interfaces has been an active area of research with significant advances. Experimental and theoretical studies involving infrared and visible excitation of adsorbed molecules resulting both in photodesorption and in light-induced chemical transformations of the adsorbate have been reported. ${ }^{1,2}$ Enhanced surface photochemistry of molecules adsorbed on surfaces capable of producing surface-enhanced Raman has been known. ${ }^{3}$ Photochemistry at surfaces may be enhanced by the same field enhancement responsible for surface-enhanced Raman scattering (SERS). ${ }^{4}$

In the observation of SERS spectra, the collection time of signals is needed, in most cases, at least $0.2 \mathrm{sec}$ or longer to get reasonable signal-to-noise ratios. Even during this time period, the enhanced surface photochemical reactions of molecules adsorbed on surfaces can possibly take place. When a single channel detector is used, there is plenty of time for the enhanced surface photochemical reactions to take place because it needs at least several minutes to scan to measure a spectrum. So far most SERS studies were done by using a single channel detector. Therefore, it is highly possible that some of published SERS spectra may be observed the photoproduct or mixture of it. ${ }^{5}$ For example, we have found the evidence of which the published SERS spectra of phthalazine were observed the photoproduct of it by using a simple flow method which is very useful to study the kinetics of relatively fast photochemical reactions of molecules adsorbed on silver nanoparticle surfaces to use SERS as a probe. $^{2}$ In this paper, we report the visible photochemical reaction of maleic acid to fumaric acid adsorbed on silver nanoparticle surface studied by a simple flow method.

\section{Experimental Section}

Silver nanoparticle solution was prepared as described previously. ${ }^{6}$ Briefly, $60 \mathrm{~mL}$ of a $2 \times 10^{-3} \mathrm{M}$ sodium borohydride solution was mixed with $22 \pm 2 \mathrm{~mL}$ of a $1 \times 10^{-3} \mathrm{M}$ silver nitrate solution. Maleic acid and fumaric acid was introduced into the silver nanoparticles as an aqueous solution. The overall concentration of maleic acid in sample solution was approximately $1.8 \times 10^{-3} \mathrm{M}$.

Poly(vinylpyrrolidone)(pvp, MW 40000) was added to the sample solution as a stabilizer, preventing further aggregation and eventual flocculation of the silver nanoparticles. The final concentration of pvp in the solution was approximately $0.027 \%$ by weight. Experiments were also carried out in the absence of pvp in order to ascertain that the polymer did not noticeably affect the spectroscopy or the kinetics.

Flow system was described previously., ${ }^{2,7}$ SERS Spectra were achieved by focused Ar ion (Lexel Model 3000) and $\mathrm{Kr}$ ion laser light (Lexel Model 3500) traversing the capillary of the flow system. SERS spectra were recorded using a Spex 1887C Triplemate spectrometer equipped with a multichannel charge-coupled device (CCD) detector. 150 spectra were collected in sequence in each flow experiment. The accumulation time of each spectrum was $5 \mathrm{sec}$. The data of the time evolution of bands were obtained from the data of 150 spectra. We made a matrix using the data of 150 
spectra. The data of each spectrum are arrayed in a column in the matrix. Consequently, the data of 150 spectra make 150 columns. In this case, the rows in the matrix correspond to the time evolution of each channel. All the Raman intensities measured by excitation of the different laser lines were calibrated by the measured $\mathrm{CCl}_{4}$ intensities. UVVisible spectra were recorded using a Varian Cary 3 UVVisible spectrometer. Pure yellowish silver nanoparticle solution was used as the reference.

\section{Flow Kinetics}

We proposed a model of the photochemical reaction which there would be two photochemical processes, isomerization and desorption, of maleic acid adsorbed on silver surfaces in our experimental conditions. ${ }^{8}$ By this model, some maleic acid may be isomerized to fumaric acid and others desorbed from the surfaces by absorption of photons.

A (maleic acid) $+n$ photons

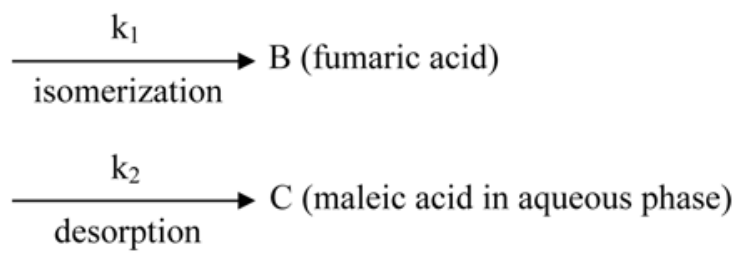

where $\mathrm{k}_{1}$ and $\mathrm{k}_{2}$ are the rate constants of the isomerization and desorption processes, respectively. The differential equations describing the rates of decrease of $\mathrm{A}$ and increase of $\mathrm{B}$ and $\mathrm{C}$ are given by

$$
\begin{aligned}
& \mathrm{d}[\mathrm{A}] / \mathrm{dt}=-\left(\mathrm{k}_{1}+\mathrm{k}_{2}\right)[\mathrm{A}] \\
& \mathrm{d}[\mathrm{B}] / \mathrm{dt}=\mathrm{k}_{1}[\mathrm{~A}] \\
& \mathrm{d}[\mathrm{C}] / \mathrm{dt}=\mathrm{k}_{2}[\mathrm{~A}]
\end{aligned}
$$

Solving these equations gives the concentrations of A, B and $\mathrm{C}$ with exposure time as

$$
\begin{aligned}
& {[A]=A_{0} \exp \left[-\left(\mathrm{k}_{1}+\mathrm{k}_{2}\right) \mathrm{t}\right]} \\
& {[\mathrm{B}]=\left[\mathrm{k}_{1} \mathrm{~A}_{0} /\left(\mathrm{k}_{1}+\mathrm{k}_{2}\right)\right]\left[1-\exp \left\{-\left(\mathrm{k}_{1}+\mathrm{k}_{2}\right) \mathrm{t}\right\}\right]} \\
& {[\mathrm{C}]=\left[\mathrm{k}_{2} \mathrm{~A}_{0} /\left(\mathrm{k}_{1}+\mathrm{k}_{2}\right)\right]\left[1-\exp \left\{-\left(\mathrm{k}_{1}+\mathrm{k}_{2}\right) \mathrm{t}\right\}\right]}
\end{aligned}
$$

where $A_{0}$ is the initial concentration of $A$, and we assumed that there is no fumaric acid at $\mathrm{t}=0$. If maleic acid needs multiphotons to react, the rate constants have these forms; $k_{1}$ $=a I^{n}, k_{2}=b I^{m}$ where $\mathrm{I}$ is the laser power used and $n$ and $m$ are the number of photons needs in each process. The letters $a$ and $b$ are the true rate constants which do not depend on the laser power used.

If the length of one dimension of a square shape laser beam is L, the exposure time of the molecules by laser beam within laser beam spot area varies from 0 (flow tail) to $A_{c} L / F$ (flow front). The sample in the laser beam spot, in the reaction zone, corresponds to the mixture of samples whose exposure time is in the range from 0 to $A_{c} L / F$. Therefore, the average concentration of maleic acid in the laser beam spot is given as

$$
\begin{aligned}
{[\overline{\mathrm{A}}] } & =\frac{1}{\mathrm{~L}} \int_{0}^{\mathrm{L}}[\mathrm{A}] d \chi \\
& =\frac{1}{\mathrm{~L}} \int_{0}^{\mathrm{L}} \mathrm{A}_{0} \mathrm{e}^{-\left(\mathrm{k}_{1}+\mathrm{k}_{2}\right) \mathrm{t}^{\prime}} d \chi \\
& =\frac{\mathrm{A}_{0} \mathrm{~h}_{0} \mathrm{~A}_{\mathrm{gc}} \mathrm{e}^{-\mathrm{t} / \tau_{0}}}{\left(\mathrm{k}_{1}+\mathrm{k}_{2}\right) \mathrm{LA}_{\mathrm{c}} \tau_{0}}\left(1-\mathrm{e}^{-\frac{\left(\mathrm{k}_{1}+\mathrm{k}_{2}\right) \mathrm{A}_{\mathrm{c}} \mathrm{L} \tau_{0} \mathrm{e}^{\mathrm{t} / \tau_{0}}}{\mathrm{~A}_{\mathrm{gc}} \mathrm{h}_{0}}}\right)
\end{aligned}
$$

where $t$ is the exposure time of laser beam. The average concentration of fumaric acid produced in the laser beam spot is also given as

$$
\begin{aligned}
& {[\overline{\mathrm{B}}]=\frac{1}{\mathrm{~L}} \int_{0}^{\mathrm{L}}[\mathrm{B}] d \chi} \\
& =\frac{\mathrm{k}_{1} \mathrm{~A}_{0}}{\left(\mathrm{k}_{1}+\mathrm{k}_{2}\right)}\left(1-\frac{\mathrm{A}_{0} \mathrm{~h}_{0} \mathrm{~A}_{\mathrm{gc}} \mathrm{e}^{-\mathrm{t} / \tau_{0}}}{\left(\mathrm{k}_{1}+\mathrm{k}_{2}\right) \mathrm{LA} \mathrm{A}_{\mathrm{c}}}\left(1-\mathrm{e}^{-\frac{\left(\mathrm{k}_{1}+\mathrm{k}_{2}\right) \mathrm{A}_{\mathrm{c}} \mathrm{L} \tau_{0} \mathrm{e}^{\mathrm{t} / \tau_{0}}}{\mathrm{~A}_{\mathrm{gc}} \mathrm{h}_{0}}}\right)\right)
\end{aligned}
$$

Therefore, the observed Raman intensity of a sample in the flow system is given as

$$
\mathrm{I}_{\mathrm{obs}}=\mathrm{I} \sigma_{\mathrm{A}}[\overline{\mathrm{A}}]+\mathrm{I} \sigma_{\mathrm{B}}[\overline{\mathrm{B}}]+\text { baseline }
$$

This equation will be used to analyze the SERS intensity of maleic acid adsorbed on silver nanoparticle surfaces.

\section{Results and Discussion}

The 2, 35, 70, 105 and 140th SERS spectra, among 150 spectra collected in sequence in a flow experiment, of maleic acid adsorbed on silver nanoparticle surfaces were shown in Figure 1 . At the early stage, the SERS spectrum was quietly different from that of the final stage. This meant that the maleic acid adsorbed silver nanoparticle surfaces was converted to fumaric acid by the irradiation of a visible laser beam. The strong band at $1391 \mathrm{~cm}^{-1}$ was due to symmetric stretching vibration of carboxylate group. The band assignments were taken from the work of Millols et al..$^{9}$ The band at $964 \mathrm{~cm}^{-1}$ was due to $\mathrm{C}-\mathrm{COO}^{-}$stretching vibration. The intensity of these bands was decreased a lot in the lower spectrum. The latter was almost disappeared. The bands at $1641 \mathrm{~cm}^{-1}$ and near $1270 \mathrm{~cm}^{-1}$ showed a different behavior. Their intensities did not change very much. The band at $1641 \mathrm{~cm}^{-1}$ was due to $\mathrm{C}=\mathrm{C}$ stretching vibration. ${ }^{10,11}$ Different exposure time of laser beam was the only possible cause of the difference in these spectra. Therefore, it was concluded that the difference was due to photochemical reaction of maleic acid on silver nanoparticle surfaces.

From our observations, it was concluded that maleic acid was isomerized to fumaric acid on silver nanoparticle surfaces by absorption of a visible laser light. Besides this photoisomerization process, there were other possible processes. When sample was irradiated by a high power of laser 


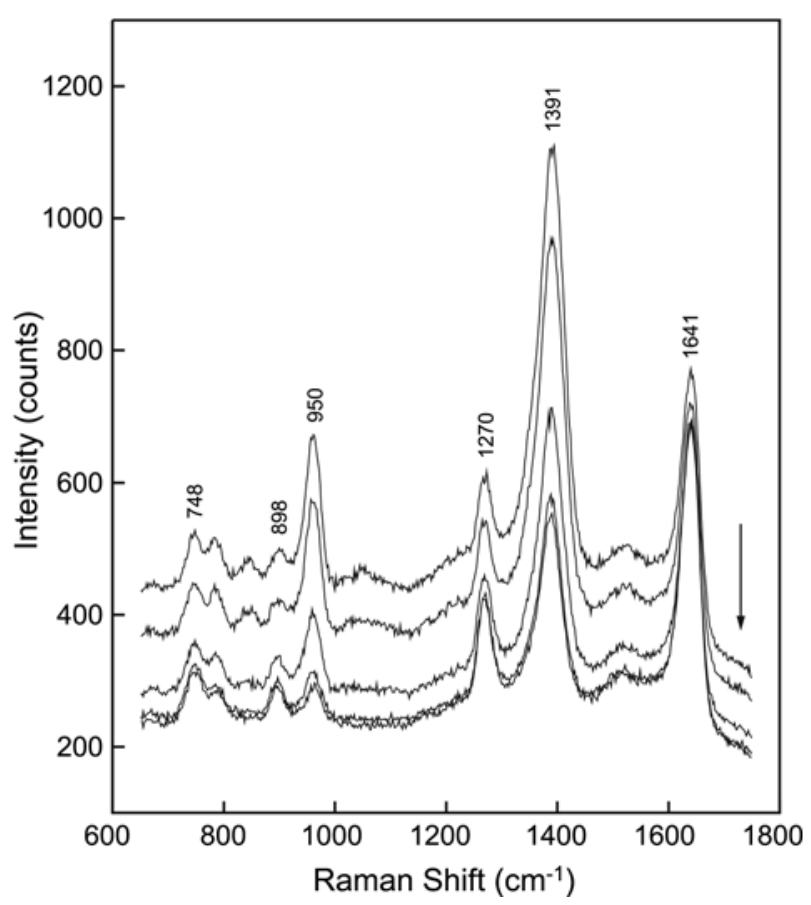

Figure 1. The 2, 35, 70, 105, and 140th SERS spectra (from top to bottom) of maleic acid adsorbed on silver nanoparticle surfaces observed using the flow system. The collection time of each spectrum was $5 \mathrm{sec}$. The excitation laser line used was excited at $530.9 \mathrm{~nm}$ and input power of $50 \mathrm{~mW}$.

light, we observed a background increasing near $1400 \mathrm{~cm}^{-1}$. This back ground is known due to graphite carbon produced by decomposition of adsorbate. ${ }^{3,4}$ In the SERS spectra of Figure 1, there was no such strong back ground. The laser powers used in these series of experiments were relatively weak. Therefore, we would not consider the photodecomposition process here. Another possible process was photodesorption of maleic acid by absorption of light. The intensity of band at $1641 \mathrm{~cm}^{-1}$ in the SERS spectra of fumaric acid was stronger than that that of maleic acid (See Figure 1). If photoisomerization was the only possible process, the intensity of the band at $1641 \mathrm{~cm}^{-1}$ should be increased in any case. Nevertheless, sometimes the intensity of the band at $1641 \mathrm{~cm}^{-1}$ was decreased in the SERS spectra of maleic acid observed by the flow method. Therefore, photodesorption was another important process to be considered.

Using the mechanistic equation derived in the previous study, ${ }^{8}$ we analyzed the SERS spectra of maleic acid observed by using the flow system. From the SERS spectra in Figure 1, we could see the change of spectrum by increasing of exposure time. For a series of laser powers, ranging from 20 to $50 \mathrm{~mW}$, of $530.9 \mathrm{~nm} \mathrm{Kr}$ ion laser line, the time evolution of the SERS intensity of the bands at 1391 and $1641 \mathrm{~cm}^{-1}$ were shown in Figures 2 and 3. The jagged lines were experimental data and the smooth lines were recalculated according to the previous equation. The fittings of two bands for the series of laser powers were done simultaneously. In fittings, we fixed the ratio of Raman cross sections of maleic and fumaric acid for each band. Without fixing this ratio in the fitting, the sum of constants $a$ and $b$

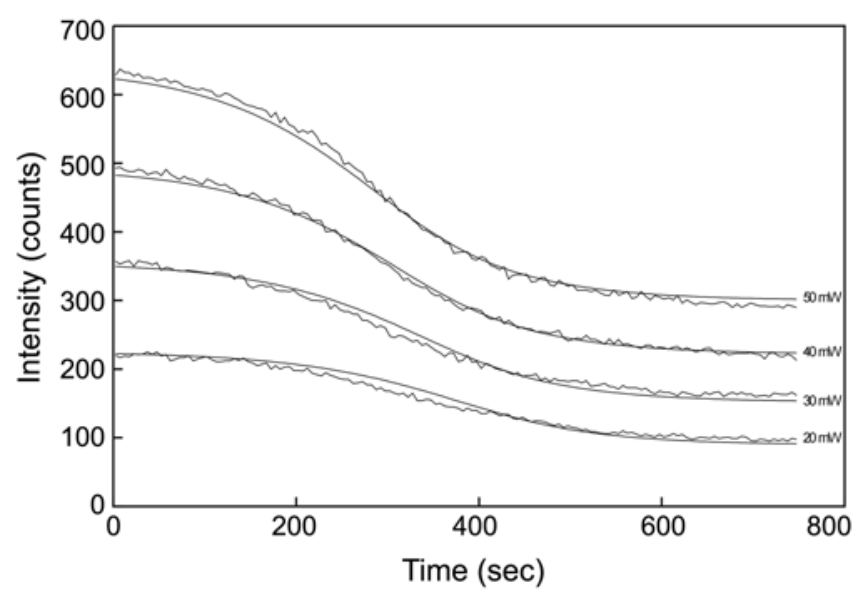

Figure 2. Time evolution of the SERS intensity at $1391 \mathrm{~cm}^{-1}$ of maleic acid adsorbed on silver nanoparticle surfaces excited with $530.9 \mathrm{~nm} \mathrm{Kr}{ }^{+}$laser line. Jagged lines are experimental data; smooth lines are recalculated according to the model described in the text. The fittings were done simultaneously according to only variation of laser beam power. The laser power from top to bottom: 50, 40, 30 , and $20 \mathrm{~mW}$.

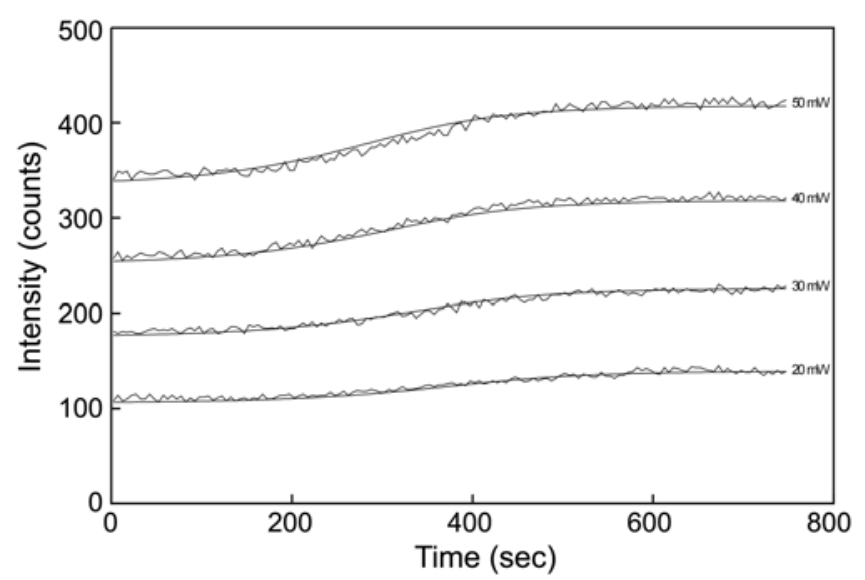

Figure 3. Same as shown in Figure 3 but for the $1641 \mathrm{~cm}^{-1}$ band.

was not varied but each value of $a$ and $b$ was varied with depending on the initial guessing values of $a$ and $b$, which these constants were related to rate constants; $k_{1}=a \mathrm{I}^{\mathrm{n}}$ and $k_{2}$ $=b \mathrm{I}^{\mathrm{m}}$. This was probably due to that $\mathrm{k}_{1}$ and $\mathrm{k}_{2}$ were not independent each other as seeing in the equations of average concentration of maleic acid and fumaric acid in the laser beam spot. Particularly, there were only $\left(k_{1}+k_{2}\right)$ terms, not $k_{1}$ or $k_{2}$ alone, in the exponential part. Therefore, for the observed SERS signals, $k_{1}, k_{2}$ and the Raman cross sections were interrelated with each other. By fixing the ration of Raman cross sections of maleic and fumaric acid for a band, the values of and $b$ were not varied with the initial guessing values of these and also the ratio of Raman cross section for the other band was conversed to a certain value in every case. For $530.9 \mathrm{~nm}$ laser line excitation, by fixing the Raman cross section ratio as 1.44 for the band at $1391 \mathrm{~cm}^{-1}$, best fittings were obtained with $a=5.9 \mathrm{sec}^{-1} \mathrm{~mW}, b=13.9 \mathrm{sec}^{-1}$ $\mathrm{mW}, n=1$ and $m=1$. The absolute value of $a$ and $b$ may not have any true meaning since the ratio of 1.44 does not base on the exact experimental data. The absolute value of $a$ and 


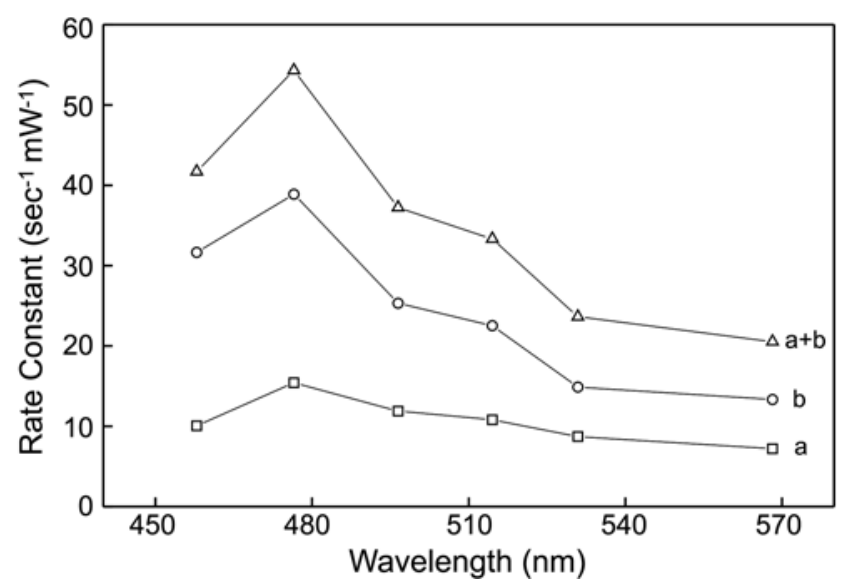

Figure 4. A comparison of the rate constants according to the wavelength of the excited laser. Constants $a$ and $b$ are from $k_{1}=a \mathbf{I}^{n}$ and $k_{2}=b \mathrm{I}^{m}$ where $n=1, m=1$, and I is the power of laser beam.

$b$ were a little different from sample to sample. The photoisomerization and phtodesorption were both one photons process $(n=1$ and $m=1)$. Photodesorption was more favorable process than photoisomerization. Both were relatively fast processes. For $50 \mathrm{~mW}$ laser power, the decay time of these processes was in the range of milli-second. Therefore, we couldn't study the kinetics of these processes by using ordinary Raman observation technique whose collection time was at least 0.2 second or longer.

The values of $a, b$ and $a+b$ measured by excitation with several laser lines are shown in Figure 4. These values increase on going toward the blue and they are at maximum near $476.5 \mathrm{~nm}$. This meant that the reaction rates of photoisomerization and desorption of maleic acid adsorbed on silver nanoparticle surfaces increased on going toward the blue. We could see the base line decreasing of SERS spectra of maleic acid when increasing the exposure time of laser beam in Figure 1. This may be related to desorption of the molecules of maleic acid from surfaces.

The total reaction rate of maleic acid on silver nanoparticle surfaces including the photodesorption and photoisomerization was at maximum near $476.5 \mathrm{~nm}$. A similar behavior was observed in studying the photochemical reaction of phthalazine adsorbed on silver nanoparticle surface. ${ }^{2}$ It is impossible to discuss the mechanism of photochemical reaction of the molecule adsorbed on metal without precise information on the energy levels of between the metal and the molecule adsorbed on metal. Unfortunately, such information was generally very limited, and maleic acid provides no exception. Anyway, the charge transfer between adsorbate and surface may involve in the surface photochemical reactions since most molecules have electronic absorption at UV or near UV but the surface photochemical reactions take place by one photon of visible light. Therefore, without any help of surface, these reactions are impossible. The reaction rate was be clearly related to the absorption of surface plasmon since it changed sample to sample whose absorption was different in each sample. Nevertheless, wavelength dependence of the reaction rate didn't follow the adsorption spectrum of surface plasmon of aggregated silver nanoparticles whose maximum was near $530.9 \mathrm{~nm}$ or longer wavelength region. Therefore, the reaction rate may also be determined by another process after surface plasmon absorption. We can consider a possible mechanism that the electrons excited by surface plasmon absorption are transferred to the excited states of the molecule adsorbed on surface and a reaction is followed. But, this proposal does not base on any solid experimental evidence. Clearly, we need more experimental data and information about the energy levels between silver and maleic acid to understand the mechanism of photochemical reaction of maleic acid adsorbed on silver nanoparticle surfaces.

\section{Conclusions}

Maleic acid adsorbed on silver nanoparticle surfaces was showed the photochemical reaction which fast desorbed from the surfaces or photoisomerized to fumaric acid by irradiation of a visible laser beam. The photoisomerization and phtodesorption were both one photons process $(n=1$ and $m=1$ ). Photodesorption was more favorable process than photoisomerization and both were relatively fast processes. It is clear that the charge transfer between adsorbate and surface involves in the surface photochemical reactions. A simple flow method used in this study is very useful to study a relatively fast photochemical reactions of molecules adsorbed on silver colloid surfaces. In addition, SERS spectra are very sensitive to detect a small quantity of photoproduct or biomaterials on metal surface to elucidate the structure mechanism of them..$^{12,13}$

Acknowledgment. N. H. Jang thanks for a partially financial support from Korea Research Foundation Grant (KRF-2003-005-C00033).

\section{References}

1. Ho, W. Surf. Sci. 1994, 299/300, 996-1007.

2. Suh, J. S.; Jang, N. H.; Jeong, D. H.; Moskovits, M. J. Phys. Chem. 1996, 100, 805-813.

3. Surface Enhanced Raman Scattering; Chang, R. K., Furtak, T. E., Eds.; Plenum: New York, 1982.

4. Blue, D.; Helwig, K.; Moskovits, M.; Wolkow, R. J. Chem. Phys. 1990, 92, 4600-4608.

5. Moskovits, M.; Suh, J. S. J. Phys. Chem. 1984, 88, 5526-5530.

6. Suh, J. S.; DiLella, D. P.; Moskovits, M. J. Phys. Chem. 1983, 87, 1540-1544.

7. Suh, J. S.; Jang, N. H.; Jeong, D. H. Bull. Korean Chem. Soc. 1994, 15, 819-821.

8. Jang, N. H.; Suh, J. S. J. Chem. Educ. 2005, submitted.

9. Millols, J.; Bardet, L.; Maury, L. J. Mol. Struct. 1974, 21, 185-195.

10. Assignments for Vibrational Spectra of Seven Hundred Benzene Derivatives; Varsanyi, G., Ed.; John Wiley \& Sons: New York, 1974; and references therein.

11. Dollish, F. R.; Fateley, W. G.; Bentley, F. F. Characteristic Raman Frequencies of Organic Compounds; John Wiley \& Sons: New York, 1974; and references therein.

12. Jang, N. H. Bull. Korean Chem. Soc. 2002, 23, 1790-1800.

13. Jang, N. H. Bull. Korean Chem. Soc. 2004, 25, 1392-1396. 